\title{
PERFORMANCE CHARACTERIZATION OF MINIATURIZED DIELECTRIC ELASTOMER ACTUATORS FABRICATED USING METAL ION IMPLANTATION
}

\author{
S. Rosset, M. Niklaus, P. Dubois, and H. R. Shea \\ Microsystems for Space Technologies Laboratory, Ecole Polytechnique Fédérale de Lausanne, \\ Switzerland
}

\begin{abstract}
We report measurements of displacement and mechanical work for miniaturized Dielectric Elastomer Actuators (DEAs) whose compliant electrodes were fabricated using metal ion implantation. 20 to $30 \mu \mathrm{m}$ thick Polydimethylsiloxane (PDMS) membranes were bonded to silicon chips with through holes of diameter 2 to $3 \mathrm{~mm}$ and were implanted on both sides with gold ions. Out-of-plane deflection recorded as a function of voltage and applied mechanical distributed load was in very good agreement with an analytical model. Unloaded vertical displacements up to $7 \%$ of the membrane's diameter were recorded and mechanical work up to $0.3 \mu \mathrm{J}$ was obtained with an applied pressure of $1 \mathrm{kPa}$. This performance data and associated model allow such miniaturized polymer actuators to be efficiently dimensioned for different applications, for instance in micropumps and active optical devices.
\end{abstract}

\section{INTRODUCTION}

Macro-scale DEAs, also known as artificial muscles, combine large displacements (strains of 100\%) with useful forces (actuation pressure of $1 \mathrm{kPa}$ ) [1]. They consist of an elastomer layer sandwiched between two compliant electrodes. In the case of free boundary conditions, an applied voltage creates a compressive force squeezing the structure, and hence expanding its area. The electrodes must be compliant enough to deform with the elastomer while remaining conductive, and are mainly made with carbon powders or greases. Although well adapted to large surfaces, carbon-based electrodes are not suitable for miniaturized DEA devices, for they require a technique to pattern the conductive electrodes on a small scale ( $\mathrm{mm}$ to $\mu \mathrm{m}$ ) while simultaneously remaining as compliant as the elastomer.

We have demonstrated the applicability of metal ion implantation by filtered cathodic vacuum arc (FCVA) to create compliant conductive layers a few nanometers below the surface of PDMS membranes, and which make suitable electrodes for miniaturized DEAs [2][3]. Unlike traditional thin-film metal deposition techniques (evaporation or sputtering), FCVA metal ion implantation has the advantage of creating a conductive layer, without forming a continuous metallic film, and thus has a low impact on the mechanical properties of the elastomer, which remains compliant.

FCVA implantation was used to create the electrodes for small-size diaphragm DEAs that were capable of outof-plane deflections of about $7 \%$ of their diameter. The recorded displacements with an external applied mechanical force were shown to be in excellent agreement with an analytical model describing the displacement of such membrane actuators when submitted to an actuation voltage and an external distributed force (pressure).

\section{DESIGN AND MODEL}

When a voltage is applied to a dielectric electroactive polymer (EAP) with free boundary conditions, it will stretch. The electrical pressure $\sigma_{e}$ generated vertically between the two electrodes is given by [4]:

$$
\sigma_{e}=-\varepsilon_{0} \varepsilon_{r} \frac{V^{2}}{t^{2}} \text {, }
$$

where $\varepsilon_{0} \varepsilon_{r}$ is the electrical permittivity of the PDMS, $V$, the applied voltage, and $t$ the PDMS thickness. Knowing the Young's modulus of the PDMS, the thickness strain $s_{z}$ can be calculated. As elastomers have a Poisson coefficient of 0.5 , the area expansion is therefore equal to $s_{z}$.

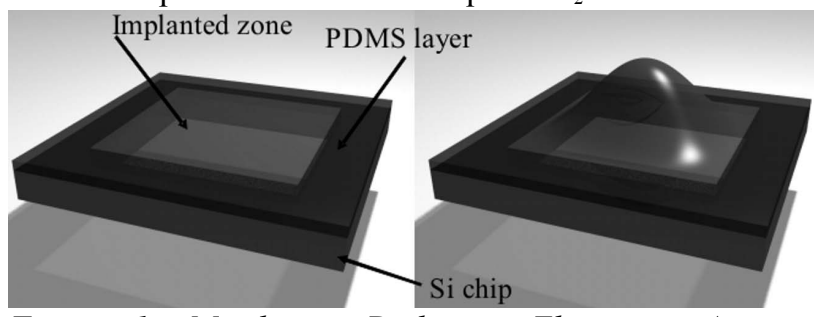

Figure 1: Membrane Dielectric Elastomer Actuator principle. Applying a voltage to the electrodes creates an electrostatic pressure which squeezes the elastomer dielectric and causes the membrane to buckle (right side).

In the case of membranes which are clamped at their circumference, area expansion is impossible, and applying a voltage will lead to compressive stress build-up in the membrane, up to the limit of stability, at which point the membrane will buckle (Figure 1). The buckling voltage $V_{b}$ is the threshold point at which out of plane deflection starts. It is a function of mechanical, electrical and geometrical properties of the EAP membrane, and for circular membranes of radius $r$ and thickness $t$, it is given by [5]:

$V_{b}=\frac{t}{\sqrt{\varepsilon_{0} \varepsilon_{r}}} \sqrt{\sigma_{0}+1.22 \frac{E}{1-v^{2}}\left(\frac{t}{r}\right)^{2}}$,

where $\sigma_{0}$ is the residual in plane stress that may be induced by the fabrication process, or voluntarily applied (prestretch). E is the Young's modulus of the compound formed by the elastomer and its two compliant electrodes, and $v$ is its Poisson coefficient. Since it is desirable to work at the lowest possible voltage, care should be taken to minimize the fabricationinduced tensile stress $\sigma_{0}$ and the impact of the electrodes on 
the PDMS Young's modulus.

A distributed force (pressure) applied on one side of the membrane creates an out of plane deformation, whose amplitude is determined by the geometrical parameters of the membrane, its Young's modulus and initial stress. The PDMS used for our actuators (Nusil CF19-2186) is very soft $(E \approx 0.5 \mathrm{MPa})$ and pressure-induced deflection can be much larger than the membrane's thickness. Consequently, the pressure-deflection relation is not dominated by bending energy, but by the stretching of the membrane, which results in a non-linear behavior. Assuming a spherical cap deformation, it is possible to derive an equation linking the deflection of the center of a circular membrane to the applied pressure [6]. Applying a voltage to a dielectric EAP which is deformed by pressure will modify its internal stress state, and hence its equilibrium position, which is therefore modulated by voltage. By inserting the voltage-dependant membrane stress into the static equilibrium equation [6], one obtains a model predicting the vertical deflection $z$ of the center of a circular membrane as a function of the applied distributed force $p$ and voltage $V$ [5]:

$$
p=(1-0.24 v)\left(\frac{8 E t}{3(1-v) r^{4}}\right) z^{3}+4\left(\frac{\left(\sigma_{0}-\varepsilon_{0} \varepsilon_{r} V^{2} / t^{2}\right) t}{r}\right) z .
$$

This model does not take into account the hyperelastic behavior of elastomers, and assumes a linear stress-strain relation, which is acceptable for strain level up to $20 \%$, as seen on macroscale uniaxial compression tests [3]. For a circular membrane deformed into a spherical cap, this $20 \%$ limit corresponds to vertical displacement up to $25 \%$ of the membrane's diameter. The model can be used for the dimensioning of buckling non-prestreched DEAs and for evaluating the influence of the main parameters on the amplitude of motion.

From the displacement $z$ of the center of the membrane that can be extracted from eq. (3), it is possible to compute the voltage-induced displacement $\Delta z$ as a function of applied pressure $p$ and voltage $V$ by subtracting the equilibrium displacement $z_{0}$ due to the applied pressure $p$ :

$$
\Delta z(p, V)=z-z_{0}=z(p, V)-z(p, 0 V) \text {. }
$$

Figure 2 illustrates the voltage-induced vertical motion computed with values representative of our actuators, and shows the influence of the two main mechanical parameters, which are determined by the fabrication process. The membrane's radius and thickness are respectively $1.5 \mathrm{~mm}$ and $20 \mu \mathrm{m}$. Plane 1) is calculated for a Young's modulus of $0.5 \mathrm{MPa}$ and a residual tensile stress of $20 \mathrm{kPa}$, which are typical values we obtain with our process for polymerized unimplanted PDMS membranes. It represents the displacements to be expected with hypothetical perfect electrodes which would not have any influence on the membrane's mechanical properties. Plane 2) is calculated for the same stress, but for a Young's modulus of $1 \mathrm{MPa}$, which typically represents the influence of ion implanted electrodes. The stiffening induced by the creation of electrodes reduces the displacement amplitude, and shows the importance of having electrodes as compliant as possible. Plane 3) is calculated for $\mathrm{E}=0.5 \mathrm{MPa}$ and a residual tensile stress of $40 \mathrm{kPa}$, which is obtained for layers polymerized at high temperature $\left(85^{\circ} \mathrm{C}\right)$. High tensile stress raises the buckling voltage and reduces the out-of plane deflection. It is therefore essential to optimize the fabrication process to minimize the in-plane stress. Some electrode fabrication methods, such as sputtering, tend to increase the tensile stress as well as having a high stiffening effect, and are thus not well adapted for DEAs [2].

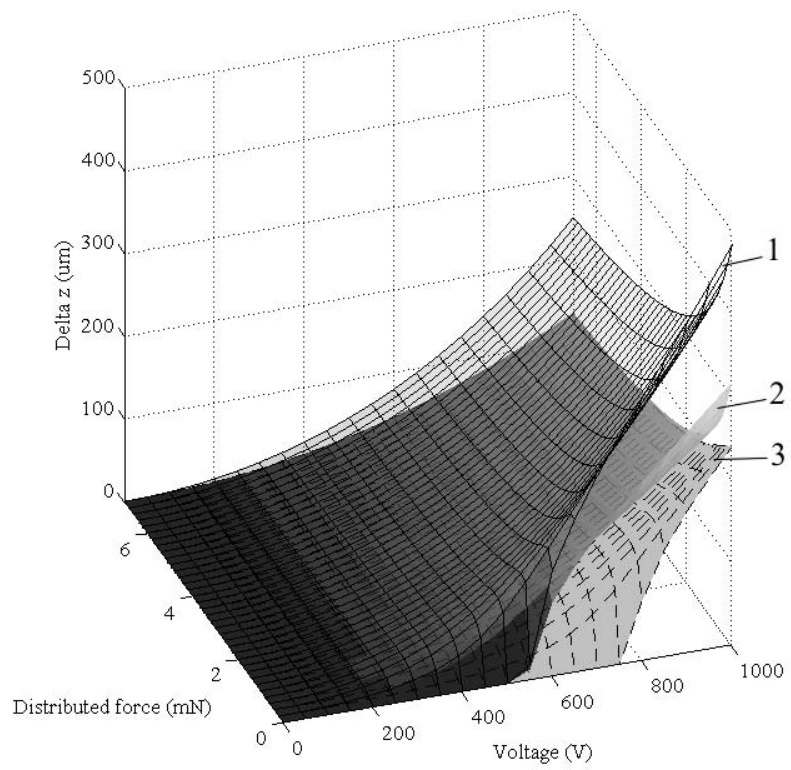

Figure 2: Voltage-induced displacement ( $\Delta z)$ calculated with the model for DEAs with different mechanical properties. 1) $E=0.5 \mathrm{MPa}, \sigma_{0}=20 \mathrm{kPa}$ 2) $E=1 \mathrm{MPa}, \sigma_{0}=20$ $\mathrm{kPa}$ 3) $E=0.5 \mathrm{MPa}, \sigma_{0}=40 \mathrm{kPa}$. Radius $1.5 \mathrm{~mm}$, thickness $20 \mu \mathrm{m}$.

The mechanical work can be calculated by integrating the pressure multiplied by the displacement of all surface elements of the membrane, over the membrane's surface.

\section{FABRICATION}

Figure 3 describes the implanted DEAs' fabrication process: a polyvinylidene chloride (PVDC) film is stretched on a rigid frame and acts as a flexible substrate on which a 4$\mu \mathrm{m}$ thick photoresist layer is applied by spin coating. Then, a mixture consisting of 58\% wt Nusil CF19-2186 PDMS and $42 \%$ wt Isooctane is spin coated on the flexible substrate between 1400 and $1600 \mathrm{rpm}$, leading to a polymerized thickness between 22 and $30 \mu \mathrm{m}$. In parallel, holes of diameter of 2 and $3 \mathrm{~mm}$ are etched through Si wafers by deep reactive ion etching (DRIE). The wafers are then diced into separate chips, which are bonded to the PDMS layer after an oxygen plasma treatment. The stack is dipped into acetone to dissolve the photoresist sacrificial layer, allowing the PVDC film to be removed. The chips, consisting of a freestanding membrane bonded on a rigid frame are implanted with a gold ion dose between $1 \cdot 10^{16}$ and $3 \cdot 10^{16}$ ions $/ \mathrm{cm}^{2}$. The topside implantation is done through a steel shadow mask to define several independent electrodes on a single chip. Metal pads 
are added on the PDMS for better electrical contact. The back side is implanted without a mask and serves as a ground plane.
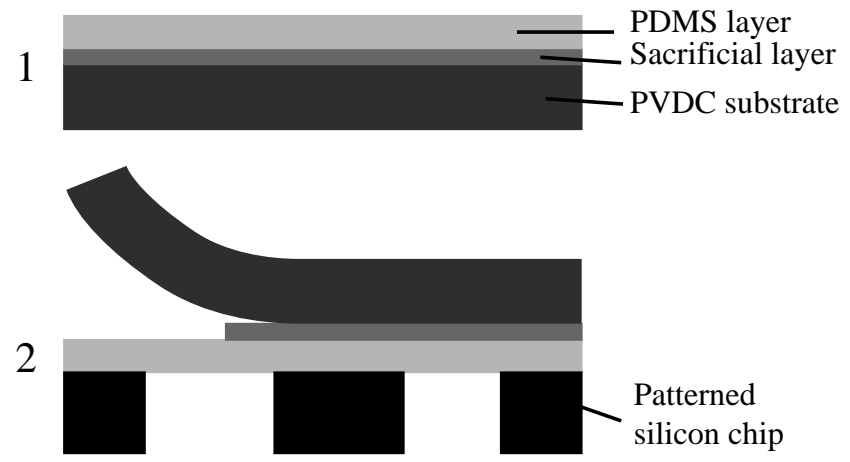

3
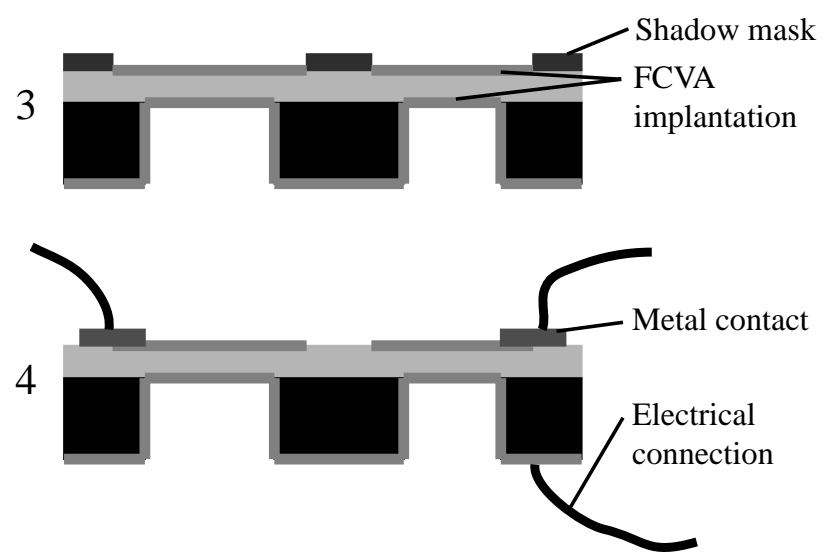

Figure 3: 1) Spinning of a sacrificial layer on a PVDC film stretched on a rigid frame, and spin coating of a 20to 30- $\mu m$ thick PDMS layer. 2) Bonding of the PDMS layer on a patterned $S i$ wafer and removal of the PVDC film by dissolution of the sacrificial layer. 3) Topside implantation through a shadow mask and backside implantation. 4) Sputtering of gold contacts and electrical connections.

\section{Implantation}

Metal ion implantation on PDMS creates a conductive layer just below the polymer's surface. By careful selection of ion dose and energy, it is possible to control the conductivity of the implanted zone, and its impact on the mechanical properties of the PDMS. For $\mathrm{Au}$ doses of $1.5 \cdot 10^{16}$ ions $/ \mathrm{cm}^{2}$ implanted by FCVA at 5 $\mathrm{keV}$, a resistivity of $120 \Omega /$ square is obtained. This is just above the percolation limit and the obtained implanted layer is conductive enough for electrostatic applications, without forming a metallic layer (e.g., the electrode is optically transparent at visible wavelengths), and thus having a limited impact on the Young's Modulus of the PDMS membrane with a relative increase of about $100 \%$. FCVA implantation is a method that is well adapted to our needs, as it produces high flux of low energy (1-10 $\mathrm{keV}$ ) ions. Low energy is necessary to limit ion penetration into the PDMS to the first 10-20 nm below the surface and high fluxes are needed to reach the desired dose $\left(1-3 \cdot 10^{16}\right.$ ions $\left./ \mathrm{cm}^{2}\right)$ in an acceptable amount of time. Most conventional implanters have very low ion flux at low energies, and require many hours of implantation to reach the targeted does. FCVA is the only implantation technique with which we have obtained compliant conducting layers that can be used to make DEAs. FCVA generates ions with several charge states. Theoretical penetration into PDMS has been simulated with SRIM [7] and is shown in Figure 4. Each ion's penetration depth is calculated as if it was penetrating unimplanted PDMS, and sputtering of PDMS and previously implanted particles by incoming ions is not taken into account.

Addition of ions at the surface of the elastomer creates a stiffening of the membrane which increases with the dose, but on another hand, ion implantation reduces the process-induced tensile stress by the inclusion of particles at the polymer's surface.

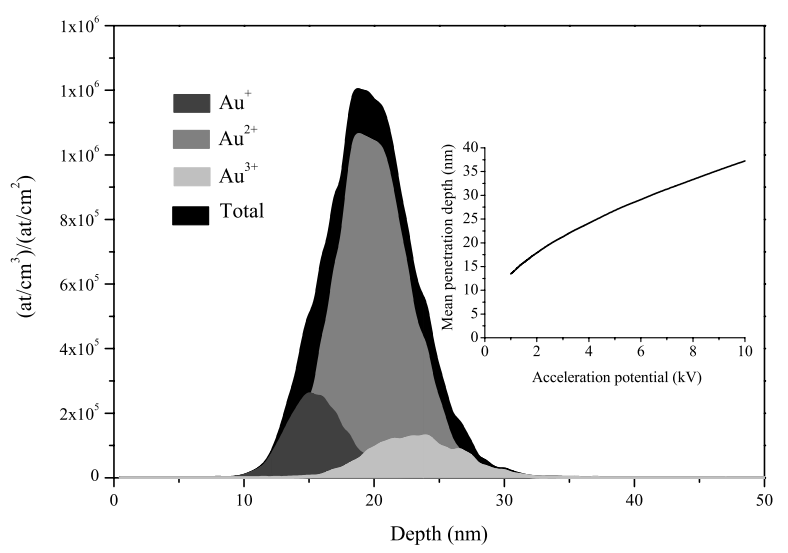

Figure 4: SRIM simulation of ion penetration for the different charge states at an acceleration potential of $2.5 \mathrm{kV}$. Inset: mean penetration of gold ions for different accelerating potentials.

\section{MEASUREMENTS}

The displacement characteristics of the actuators were measured by mounting the chips on an airtight socket. A differential air pressure between 0 and $1 \mathrm{kPa}$ in steps of $20 \mathrm{~Pa}$ was applied on one side of the membrane with a syringe pump, and its deformation was measured with an optical profiler (Wyko NT1100). The first measurement is made without any applied voltage on the electrodes. It is a so-called bulge test measurement, and it allows the determination of the Young's modulus and residual stress of the tested membrane [6]. Additional measurements are performed for different applied voltage values up to the dielectric breakdown of the elastomer. Displacement versus applied pressure and voltage can be plotted and compared to the theoretical model fitted with the mechanical parameters obtained with the bulge test measurement (Figure 5). Data points are in good agreement with the theory. Discrepancies are observed when there is no external force applied. The buckling threshold is not observed on the measured ponts, and vertical displacement occurs even at voltages below $V_{b}$. This is due to initial deformation of the membrane, which is not perfectly flat. Small deformations in the PDMS membrane can promote premature buckling by creating an out-of-plane force component. The DEA whose performance is reported in Figure 5 is heavily implanted $\left(2 \cdot 10^{16}\right.$ ions $\left./ \mathrm{cm}^{2}\right)$, which leads to a low residual stress of 10 $\mathrm{kPa}$ (and therefore a low buckling voltage), but a high 
Young's modulus 5.7 times higher than that of the membrane before implantation. As a result, the maximum voltage-induced displacement is $150 \mu \mathrm{m}$, or $5 \%$ of the membrane's diameter. With less implanted membranes, maximal voltage-induced displacement of up to $7 \%$ of the membrane's size has been measured [3].

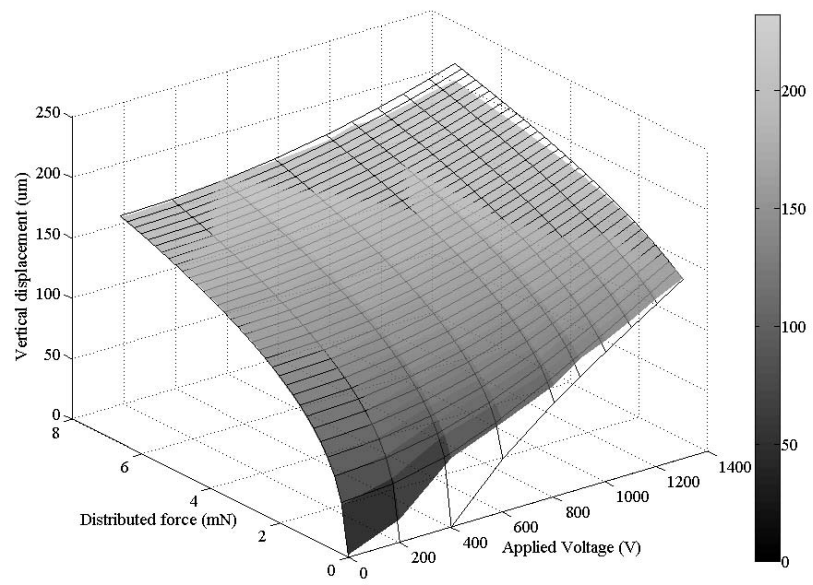

Figure 5: Measured (plane) and theoretical (wireframe) displacement for a 3mm diameter, $24.5 \mu \mathrm{m}$ thick DEA.

With increasing external force, the voltage-induced displacement decreases. For the maximal distributed force of $6.6 \mathrm{mN}$ (corresponding to a pressure of $934 \mathrm{~Pa}$ ), the maximal $\Delta z$ obtained was reduced to $32 \mu \mathrm{m}$. This is however the point where the mechanical work is maximized with a value of $107.5 \mathrm{~nJ}$. On less implanted membranes, mechanical work up to $300 \mathrm{~nJ}$ were obtained (Figure 6).

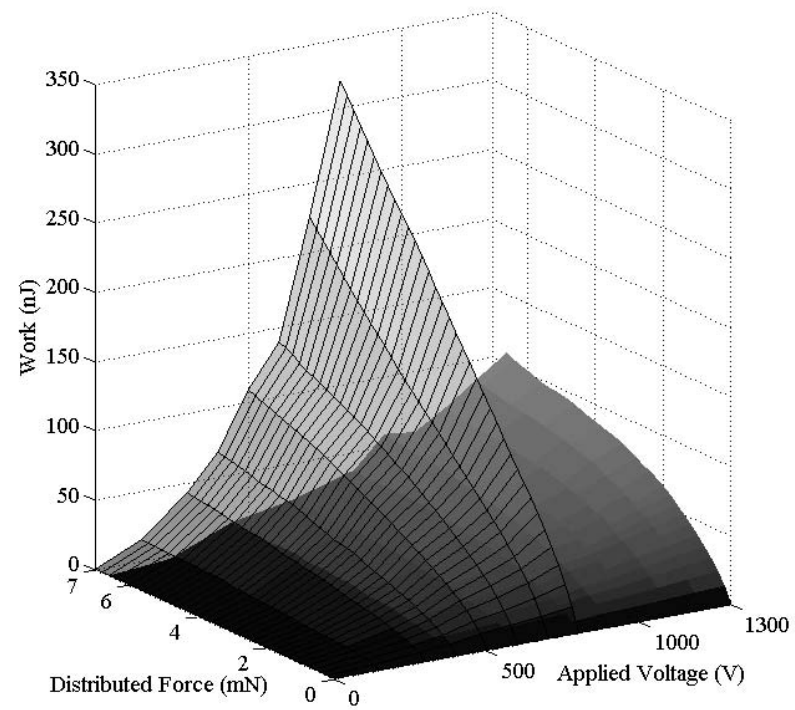

Figure 6: Mechanical work obtained from a DEA implanted with $1 \cdot 10^{16} \mathrm{at} / \mathrm{cm}^{-2}$ (wireframe) and $2 \cdot 10^{16}$ at $\mathrm{cm}^{-2}$ (plane)

\section{CONCLUSIONS}

Metal ion implantation is a promising technique to create compliant electrodes for $\mu \mathrm{m}$ - to mm-size DEA. Our ionimplanted DEAs can achieve large unloaded vertical displacement of $5-10 \%$ of their lateral size and can perform mechanical work that allows displacing loads in the $\mathrm{mN}$ range by several micrometers. Out-of-plane deflection of diaphragm actuator can be predicted by an analytical model for displacement up to $25 \%$ of the membrane's diameter and strongly depends on the mechanical properties of the active membrane, which are in turn linked to the implantation parameters. The optimal dose for ion implanted electrodes is a tradeoff between conductivity and modification of the mechanical properties of the dielectric layer. Too high a dose creates low-resistivity electrodes, but stiffens the elastomer resulting in a reduced displacement capability. Too low a dose leads to highly compliant conducting layers but whose resistivity is unstable.

Ion implanted electrodes also have the advantage of being transparent to visible light, which opens up the field of using DEAs for optical applications for which transmission is desirable.

\section{ACKNOWLEDGEMENTS}

The authors wish to thank the COMLAB staff for help with device fabrication, and acknowledge financial support from the Swiss National Science Foundation grant \#20021111841 and from the EPFL.

\section{REFERENCES}

[1] S. Ashley, “Artificial Muscles", Scientific American, vol. 289, pp. 52-59, 2003.

[2] S. Rosset, M. Niklaus, P. Dubois, M. Dadras, and H. Shea, "Mechanical properties of electroactive polymer microactuators with ion-implanted electrodes", in Proceedings of SPIE, vol. 6524, 2007.

[3] P. Dubois, S. Rosset, S. Koster, J. Stauffer, S. Mikhailov, M. Dadras, N.-F. de Rooij, and H. Shea, "Microactuators based on ion implanted dielectric electroactive polymer (EAP) membranes", Sensors and Actuators A: Physical, vol. 130-131, pp. 147-154, 2006.

[4] R. E. Pelrine, R. D. Kornbluh, and J. P. Joseph, "Electrostriction of polymer dielectrics with compliant electrodes as a means of actuation", Sensors and Actuators A: Physical, vol. 64, pp. 77-85, 1998.

[5] S. Rosset, M. Niklaus, P.Dubois, and H. R. Shea, "Mechanical Characterization of a Dielectric Elastomer Microactuator With Ion-Implanted Electrodes", submitted to Sensors and Actuators A: Physical, 2007.

[6] B. E. Alaca, J. C. Selby, M. T. A. Saif, and H. Sehitoglu, "Biaxial testing of nanoscale films on compliant substrates: Fatigue and fracture", Review of Scientific Instruments, vol. 73, pp. 2963-2970, 2002.

[7] SRIM: SRIM - The Stopping and Range of Ions in Mattersoftware at: http://www.srim.org/ 